\title{
ESTUDO DAS ANOMALIAS CROMOSSÔMICAS OCORRIDAS EM UMA MATERNIDADE NOS ANOS DE 2010 A 2014
}

\author{
Cleiton Fantin ${ }^{1}$, Marcelo Lasmar Santos ${ }^{2}$, Lilian Barroso Carvalho², Natanael Martins Gomes², Larissa \\ Nascimento Souza ${ }^{2}$, Gabriela Bentes Sousa ${ }^{3}$
}

\begin{abstract}
RESUMO: O presente estudo buscou identificar a existência de anomalias cromossômicas registradas nos prontuários de nascidos vivos em uma maternidade. Estudo retrospectivo que analisou as informações contidas nos prontuários dos arquivos do Serviço de Arquivamento Médico de uma maternidade do estado do Amazonas entre janeiro de 2010 e dezembro de 2014, e estudou-se a correlação de anomalias cromossômicas presentes com características maternas e do nascido vivo. Analisou-se 15.621 prontuários, destes 163 apresentaram defeitos congênitos, 15 foram diagnosticados com síndromes cromossômicas distribuídas em três tipos de anomalias: 13 indivíduos com Síndrome de Down, um com Síndrome de Patau e um com Síndrome de Dany-Walker. Este é o primeiro registro de ocorrência e perfil dos nascimentos com anomalias cromossômicas em uma maternidade. O resultado é de grande importância para a saúde pública do Estado. A realização de novos estudos poderá fornecer um melhor panorama sobre diferentes doenças genéticas daquele estado.

DESCRITORES: Anormalidades congênitas; Gravidez; Epidemiologia descritiva; Nascimento vivo.
\end{abstract}

\section{STUDY OF CHROMOSOMAL ABNORMALITIES ENCOUNTERED IN A MATERNITY HOSPITAL BETWEEN 2010 AND 2014}

\begin{abstract}
This study sought to identify the existence of chromosomal abnormalities recorded in the medical records of live-born infants in a maternity hospital. This retrospective study analyzed the information contained in the medical records of the archives of the Medical Archiving Service of a maternity hospital in Amazonas state between January 2010 and December 2014, with the correlation between the chromosomal abnormalities and the characteristics of the mothers and the live-born infants also studied. A total of 15,621 records were analyzed, of these 163 presented congenital defects, with 15 diagnosed with chromosomal syndromes, divided into three types of anomalies: 13 individuals with Down syndrome, one with Patau syndrome and one with Dandy-Walker syndrome. This is the first registration of the occurrence and profile of births with chromosomal abnormalities in a maternity hospital. The result is of great importance for the public health service of the state. The performance of further studies may provide a better overview of the different genetic diseases of this state.

DESCRIPTORS: Congenital abnormalities; Pregnancy; Descriptive epidemiology; Live-born infant.
\end{abstract}

\section{ESTUDIO DE LAS ANOMALÍAS CROMOSÓMICAS QUE OCURRIERON EN UNA MATERNIDAD EN LOS AÑOS DE 2010 A 2014}

RESUMEN: Este estudio tuvo la finalidad de identificar la existencia de anomalías cromosómicas registradas en los prontuarios de nacidos vivos en una maternidad. Estudio retrospectivo hecho por medio de análisis de informaciones contenidas en los prontuarios de los archivos del Servicio de Archivo Médico de una maternidad del estado de Amazonas entre enero de 2010 y diciembre de 2014. Fue examinada la correspondencia de anomalías cromosómicas presentes con características maternas y del nacido vivo. Se analizaron 15.621 prontuarios, de los cuales 163 presentaron defectos congénitos, 15 fueron diagnosticados con síndromes cromosómicas distribuidas en tres tipos de anomalías: 13 individuos con Síndrome de Down, un con Síndrome de Patau y un con Síndrome de Dany-Walker. Este es el primero registro de ocurrencia y perfil de los nacimientos con anomalías cromosómicas en una maternidad. El resultado tiene gran importancia para la salud pública del estado. La realización de nuevos estudios podrá traer un mejor panorama acerca de distintas enfermedades genéticas del estado.

DESCRIPTORES: Anormalías congénitas; Gravidez; Epidemiología descriptiva; Nacimiento vivo.

${ }^{1}$ Biólogo. Doutor em Biotecnologia. Docente do Programa de Pós-Graduação em Biotecnologia e Recursos Naturais da Amazônia da Universidade do Estado do Amazonas. Manaus, AM, Brasil.

${ }^{2}$ Discente de Medicina. Universidade do Estado do Amazonas. Manaus, AM, Brasil.

${ }^{3}$ Médica. Residente de Ginecologia e Obstetrícia da Universidade do Estado do Amazonas. Manaus, AM, Brasil.

Autor Correspondente:

Recebido: 23/09/2016

Cleiton Fantin

Universidade do Estado do Amazonas

Finalizado: $20 / 02 / 2017$

Av. Carvalho Leal, 1777 - 69065-001 - Manaus, AM, Brasil

E-mail:cfantin@uea.edu.br 


\section{INTRODUÇÃO}

Durante o desenvolvimento embrionário, alterações podem ocorrer resultando no aparecimento de anomalias congênitas, causando o aparecimento de pequenas assimetrias ou até mesmo defeitos que podem comprometer a estética e funcionalidade ${ }^{(1-2)}$.

O índice de anomalias congênitas chega à marca de 2 a 3\% entre os nascidos vivos, acometendo diferentes órgãos e sistemas. Calcula-se que $25 \%$ dessas malformações estejam relacionadas à genética, $10 \%$ de procedência ambiental e $65 \%$ seriam causadas por fatores de ordem desconhecida ${ }^{(3)}$.

Segundo estudos ${ }^{(4)}$, todas as populações estão expostas aos riscos de desenvolverem malformações congênitas, entretanto, a frequência e o tipo destas malformações variam com a raça, a etnia e as condições socioeconômicas, assim como o acesso aos serviços de saúde, a nutrição, o estilo de vida e a educação materna, particularmente às relacionadas ao tubo neural ${ }^{(4)}$.

Com avanço da tecnologia, alguns testes para detecção de defeitos congênitos são realizados durante a gravidez, levando a uma melhora significativa no poder de diagnóstico para diversas patologias congênitas e anomalias do desenvolvimento. O estudo citogenético e os ensaios enzimáticos permitem o diagnóstico da maioria das anomalias cromossômicas e erros inatos de metabolismo no período pré-natal ${ }^{(5)}$.

Apesar dos testes serem precisos, muitos dos casos de síndromes cromossômicas não são identificados durante a vida fetal, já que o teste específico para tal detecção é aplicado principalmente em mães com mais de 35 anos. Assim, os registros demonstram que é superior a percentagem de crianças com síndromes cromossômicas cujas mães possuem menos de 30 anos $^{(6)}$.

Quando são realizados os exames do pré-natal, conclui-se que as aneuploidias estão entre as anormalidades genéticas mais comuns, sendo diagnosticadas com maior frequência as trissomias que envolvem os cromossomos 13, 18 e 21. Entre as trissomias mais frequentes observadas ao nascimento, podemos observar a Síndrome de Down (trissomia do cromossomo 21), precedida pela Síndrome de Edwards (trissomia do 18) ${ }^{(7)}$.

O avanço da genética, nas últimas décadas, permitiu novas descobertas em relação às diversas doenças genéticas. Contudo, em se tratando de anomalias cromossômicas na região norte do Brasil, muito ainda é necessário a se reportar, como, por exemplo, alguns aspectos básicos como seus dados epidemiológicos e estatísticos. O estímulo à elaboração deste trabalho foi a escassez de dados a respeito do levantamento das anomalias cromossômicas no estado do Amazonas.

Sendo assim, o objetivo deste estudo foi identificar as anomalias cromossômicas ocorridas em nascidos vivos em uma maternidade de referência da cidade de Manaus e, quando possível, associar fatores relacionados ao perfil materno com o dos nascidos vivos que apresentaram determinada anomalia cromossômica.

\section{MÉTODO}

A presente pesquisa é um estudo retrospectivo com caráter descritivo(8) que propôs analisar a prevalência de crianças nascidas vivas com anomalias cromossômicas no período de janeiro de 2010 a dezembro de 2014 em uma maternidade na cidade de Manaus-AM, Brasil.

A população estudada foi composta por registros de crianças nascidas vivas com diagnóstico clínico e/ou citogenético de anomalias cromossômicas no período de janeiro de 2010 a dezembro de 2014. Entre os meses de fevereiro a setembro de 2015, foram analisados 15.621 prontuários de crianças nascidas vivas no período de janeiro de 2010 a dezembro de 2014, arquivados na sala do Serviço de Arquivamento Médico (SAME) da maternidade, para identificar a presença de anomalias cromossômicas descritas nos mesmos.

Como critério de inclusão, foram analisados todos os prontuários com casos de malformações congênitas. Foram excluídos os recém-nascidos que não apresentavam diagnósticos clínico e/ 
ou laboratorial de malformação congênita, e prontuários com preenchimento incorreto ou que apresentaram inconsistência das informações, não encontrados pela equipe de pesquisa ou danificados (molhados, manchados, entre outros).

Para coleta dos dados, foi utilizado preenchimento de questionário estruturado embasado em informações contidas na Declaração de Nascidos Vivos (DNV), bem como fichas de preenchimento médico realizada por obstetras, neonatologistas, intensivistas e, quando presente, informações contidas em exames de diagnóstico e imagem como ultrassonografias e radiografias.

Com o preenchimento do questionário/instrumento, foi possível gerar dados gerais e específicos quanto à apresentação dos recém nascidos no período pré e pós natal, referente a qualquer alteração congênita relatada pelo médico assistencialista. Dentre as informações, destacam-se aquelas referentes às características maternas como: idade materna, histórico de má formação congênita familiar e abortos, tipo de parto, realização e número de consultas pré-natal; relacionadas ao nascido vivo: sexo, peso, índice de Apgar no $1^{\circ}$ e $5^{\circ}$ minuto e idade gestacional.

Após coleta, os dados foram inicialmente tabulados em um banco de dados no programa da Microsoft Office Excel 2013 conforme as variáveis analisadas e posteriormente transferidas para o software estatístico SPSS 16.0 e realizados testes para obtenção de média, mediana e desvio padrão.

O estudo foi submetido e aprovado pelo Comitê de Ética em Pesquisa da Universidade do Estado do Amazonas sob o Parecer n. 048/2012, sendo respeitados os aspectos éticos e legais de pesquisas que envolvem seres humanos.

\section{RESULTADOS}

Mediante análise dos prontuários, foram registrados 15.621 nascimentos; destes, 163 (1,04\%) apresentaram malformações congênitas, dos quais 81 (49,70\%) eram do sexo masculino e 72 (44,17\%) eram do sexo feminino, outros $10(6,13 \%)$ foram casos de sexo indeterminado, ignorado ou sem informação.

Diferentes tipos de malformações congênitas foram encontrados entre os nascidos vivos, em que as anomalias cromossômicas representaram 15 (9,20\%), sendo as do aparelho urinário menos frequentes, duas $(1,22 \%)$, e as do sistema nervoso mais frequentes, $37(22,70 \%)$.

Dentre as 15 anomalias cromossômicas encontradas nos prontuários analisados, estavam registradas três tipos de síndromes: Síndrome de Down 13 (86,66\%), Síndrome de Patau uma (6,67\%) e Síndrome de Dany-Walker uma (6,67\%). Dados sobre o perfil dos nascidos vivos que apresentaram anomalias cromossômicas encontram-se na Tabela 1. Em relação à mãe, também foram analisados dados do perfil materno, com base em dados de 13 mulheres que realizaram o pré-natal, apresentados na Tabela 2.

Tabela 1- Perfil dos nascidos vivos que apresentaram anomalias cromossômicas no período de 2010-2014, na Maternidade Balbina Mestrinho. Manaus, AM, Brasil, 2016

\begin{tabular}{|c|c|c|c|c|c|c|c|c|c|c|c|c|c|c|}
\hline \multirow[t]{2}{*}{ Anomalia } & \multirow[t]{2}{*}{$\mathbf{N}$} & \multicolumn{2}{|c|}{ Sexo } & \multicolumn{2}{|c|}{ Apgar $1^{\circ} \mathrm{min}$} & \multicolumn{2}{|c|}{ Apgar $5^{\circ} \mathrm{min}$} & \multicolumn{3}{|c|}{ Idade gestacional } & \multicolumn{4}{|c|}{ Peso } \\
\hline & & $\mathbf{M}$ & $\mathbf{F}$ & $<7$ & $>7$ & $<7$ & $>7$ & $<37$ & 37 a 41 & $>42$ & B & I & A & MF \\
\hline SD & 13 & 4 & 9 & - & 13 & - & 13 & 1 & 10 & 2 & 1 & 4 & 7 & 1 \\
\hline SP & 1 & - & 1 & 1 & - & 1 & - & 1 & - & - & 1 & - & - & - \\
\hline SDW & 1 & - & 1 & - & 1 & - & 1 & 1 & - & - & - & 1 & - & - \\
\hline
\end{tabular}

Legenda: $\mathrm{SD}=$ Síndrome de Down, $\mathrm{SP}=$ Síndrome de Patau, SDW= Síndrome de Dany-Walker, M=masculino, F=feminino, $\mathrm{B}=$ baixo, $\mathrm{I}=$ insuficiente, $\mathrm{A}=$ adequado $\mathrm{MF}=$ macrossomia fetal. 
Tabela 2 - Dados maternos de mães que apresentaram filhos nascidos vivos com anomalias cromossômicas ocorridas no período de 2010-2014, na Maternidade Balbina Mestrinho. Manaus, AM, Brasil, 2016

\begin{tabular}{|c|c|c|c|c|c|c|c|c|c|c|c|c|c|c|c|}
\hline \multirow{2}{*}{$\begin{array}{l}\text { Ano- } \\
\text { malia }\end{array}$} & \multirow[t]{2}{*}{$\mathbf{N}$} & \multicolumn{5}{|c|}{ Idade materna } & \multicolumn{2}{|c|}{ CMF } & \multicolumn{2}{|c|}{ Hist.de aborto } & \multicolumn{2}{|c|}{ Parto } & \multicolumn{3}{|c|}{ Numero de consultas } \\
\hline & & $\begin{array}{c}15 a \\
19 \\
\end{array}$ & $\begin{array}{c}20 \mathrm{a} \\
24\end{array}$ & $\begin{array}{c}25 a \\
29 \\
\end{array}$ & $\begin{array}{c}30 \text { a } \\
34 \\
\end{array}$ & $\geq 35$ & SI & $\mathbf{N}$ & $\mathrm{S}$ & $\mathbf{N}$ & V & $\mathrm{C}$ & 1 a 3 & 4 a 6 & $\geq 7$ \\
\hline SD & 13 & 4 & 1 & 2 & 1 & 4 & 10 & 3 & 2 & 11 & 9 & 4 & 2 & 7 & 3 \\
\hline SP & 1 & - & - & - & 1 & - & - & 1 & 1 & - & - & 1 & - & 1 & - \\
\hline SDW & 1 & - & 1 & - & - & - & 1 & - & - & 1 & - & 1 & - & - & 1 \\
\hline
\end{tabular}

Legenda: $\mathrm{SI}=$ sem informação, $\mathrm{V}=$ vaginal, $\mathrm{C}=$ cesáreo, (-) nenhum caso registrado. $\mathrm{SD}=$ Síndrome de Down, $\mathrm{SP}=\mathrm{Síndrome} \mathrm{de}$ Patau, SDW= Síndrome de Dany-Walker. S=sim, N=não, CMF=Caso de malformação familiar.

Itálico= caso de SD faixa etária materna SI; 1 caso de SD número de consultas pré-natal Ignorado.

\section{- DISCUSSÃO}

Quando se trata de doenças genéticas, sabemos que os distúrbios cromossômicos estão comumente encontrados nos abortamentos, malformações congênitas e retardos do desenvolvimento. A causa dessas anomalias está relacionada principalmente com posição, localização e número de genes distribuídos no material genético ${ }^{(9)}$. Alguns estudos relatam a prevalência de nascidos vivos com anomalias congênitas variando entre $1,0 \%$ e $7,0 \%(10)$.

Os resultados do presente estudo encontraram a incidência de $163(1,04 \%)$ de nascidos vivos com anomalias congênitas, dados semelhantes ao encontrado por outros estudos realizados em maternidades brasileiras. No estudo da maternidade no município de Campina Grande-PB ${ }^{(11)}$, o resultado foi de 1,1\% de nascidos vivos com anomalias congênitas, baseado na busca nos prontuários obstétricos e neonatais. Resultado semelhante com 1,2 \% dos nascidos vivos de uma maternidade de Porto Alegre-RS ${ }^{(12)}$ e 1,3\% reportados em uma maternidade de Campinas-SP ${ }^{(13)}$.

Valores menores do que o encontrado em nossos resultados foram apontados em um estudo dos nascimentos de mães residentes no município de Maringá-PR, que registrou $0,8 \%$ de casos de nascidos vivos com algum tipo de anomalia congênita ${ }^{(14)}$ e os $0,8 \%$ reportados para a cidade do Rio de Janeiro ${ }^{(15)}$. No entanto, estudos também demonstram valores ainda menores nesta incidência, como os 0,4\%, encontrado pela Secretaria de Saúde de Contagem em Minas Gerais ${ }^{(16)}$.

Diversos fatores podem gerar anomalias congênitas, e estas estão diretamente relacionadas a eventos que precedem ao nascimento, podendo ser de origem hereditária ou adquirida ${ }^{(1)}$. Os defeitos cromossômicos podem ser decorrentes de gametas geneticamente anormais e de irregularidades na divisão do embrião. Existe a prevalência de alterações cromossômicas relacionadas com o gameta feminino, e isto está intimamente relacionado com o fato dos óvulos apresentarem a idade cronológica da mulher, diferentemente dos espermatozoides, que são renovados a cada dois meses ${ }^{(17)}$.

Em relação à anomalia cromossômica mais frequente, em nossos estudos a incidência de Síndrome de Down nos nascidos vivos na maternidade estudada, foi a anomalia cromossômica mais frequente $13(86,66 \%)$, o que está de acordo com o disposto em literatura, e foi seguida de apenas duas outras síndromes (Síndrome de Patau e Síndrome de Dany-Walker). No Brasil anualmente existem em média 8.000 nascidos vivos com SD e a incidência é de 600/800 nascimentos. Dados demonstram que, dentre os deficientes mentais assistidos em instituições especializadas brasileiras, cerca de 18-20\% são SD ${ }^{(18)}$.

É fato que entre as aneuploidias, as mais frequentes estão relacionadas com a ocorrência de um cromossomo autossômico adicional, ou trissomia (três cromossomos, ao invés do par normal). Dentre elas, o tipo mais comum de aneuploidia humana viável é a Síndrome de Down, ou trissomia do 21, que pode ser resultante da não-disjunção meiótica (meiose I ou II) do par cromossômico $21^{(19)}$.

A idade reprodutiva da mãe tem sido um dos fatores relacionados ao surgimento das trissomias cromossômicas em humanos, quanto mais avançada a idade, maior será o risco ${ }^{(20)}$. De fato, isto foi demonstrado na Bahia, com um estudo retrospectivo relacionando idade materna e Síndrome de Down, 
que demonstrou a correlação de 43,6\% entre idade das mães ao parto e nascidos com Síndrome de Down $^{(3)}$. Em nossos estudos, quando analisado o fator de idade materna, relacionados com o número de nascidos com anomalias cromossômicas, encontramos prevalências iguais entre mulheres de 15-19 e $>35$ anos, $4(26,66 \%)$.

Em outro estudo ${ }^{(20)}$ demonstrou-se que em mães adolescentes com gestações múltiplas, a probabilidade de gerar uma criança com anomalia cromossômica era de 6,1 vezes comparados a adolescentes com gestação única, e para as mães tardias, as chances são de 11,4 quando comparadas as mães com idade entre 20 a 34 anos. Em contrapartida, existem alguns estudos realizados no Brasil que não apontaram associação entre idade materna e anomalia congênita ${ }^{(21-22)}$. O decréscimo do número de óvulos e embriões aneuploides em mulheres com idade mais elevada também pode estar relacionado com o surgimento das trissomias ${ }^{(2)}$.

Por esta razão, o exame de cariótipo realizado ainda no feto tem sido recomendado pra mães com idade avançada. Desde a década de 80, os centros de diagnósticos em países desenvolvidos já realizam exames para detectar esse tipo de anomalia, principalmente a Síndrome de Down.

O exame de cariótipo é uma ferramenta importante, esclarecedora e significante para casais que necessitam de diagnóstico para o seu recém nascido. Com este exame, é possível identificar diferentes tipos de anomalia cromossômica, como aquelas decorrentes de translocação ou inversão cromossômica. Além de fornecer o diagnóstico correto e conclusivo, a informação gerada é importante para o médico clínico/responsável estabelecer o risco de outras perdas gestacionais ou malformações fetais nas gestações futuras, ou no caso de casais com cariótipo normal, direcionar as investigações para outras causas genéticas ou não genéticas, conforme protocolos estabelecidos na literatura ${ }^{(19)}$.

O diagnóstico definitivo de anomalias congênitas é de fundamental importância para os pais cujos filhos são portadores de alguma anomalia. Apesar da frequência baixa, o diagnóstico definitivo promove o adequado e efetivo aconselhamento genético e reprodutivo, corroborando no provável sucesso perante uma nova gravidez ${ }^{(23)}$.

De acordo com os resultados das análises, a maioria das gestantes realizou o exame pré-natal. É de extrema importância que se saiba o quadro clínico e o prognóstico dos pacientes com anomalias cromossômicas, pois essa situação irá ajudar a decidir a estabelecer ou não tratamentos invasivos, como por exemplo intervenções cirúrgicas. Torna-se de grande importância diagnósticos confirmados rapidamente para que a conduta médica possa tomar decisões corretas.

Um bom exemplo da aplicabilidade da importância de realização do pré-natal, diz respeito à identificação dos pacientes que apresentam gestação com trissomia do cromossomo 18, principalmente para antecipar os preparos para o nascimento. É válido ressaltar que, devido ao Art. 128 do Código Penal - Decreto Lei 2848/40 vigente no Brasil, não é permitida a interrupção da gestação, mesmo quando o diagnóstico de anomalia cromossômica é confirmado, existe exceção para apenas dois casos onde a gravidez poderá ser interrompida: primeiro pela de provável morte da mãe ou em casos de violência sexual ${ }^{(21)}$.

Muitos casais podem ser portadores de alterações cromossômicas e irão transmiti-las a seus descendentes. Para estes casais que apresentam alterações cromossômicas, durante a realização do processo de aconselhamento genético, seria muito importante a indicação para a realização de Diagnóstico Pré-Natal (vilosidades coriônicas ou amniocentese) caso o casal opte por outras gestações. Assim como informar da possibilidade de uma reprodução assistida acompanhada pelo Diagnóstico Genético Pré-Implantação (PGD) para a seleção de embriões não portadores de anomalias cromossômicas, antes de sua implantação ${ }^{(19)}$.

É importante que o profissional de saúde tenha em mente que o Aconselhamento Genético não envolve conhecimentos inacessíveis e pode ser realizado onde não há geneticista ${ }^{(23)}$. Quando não há geneticista disponível, outro profissional de saúde pode ser treinado para receber as informações necessárias para poder realizar o aconselhamento genético, mesmo que este aconselhamento seja de maneira simples, para dar informações básicas ao casal, que geralmente não dispõe de informação alguma sobre hereditariedade.

O aconselhamento genético ideal seria aquele realizado com anamnese minuciosa, exame 
clínico detalhado, fotos de corpo inteiro e da região afetada, no caso de anomalias físicas, estudo anatomopatológico, no caso de óbito, raios $\mathrm{X}$ de esqueleto nos casos de suspeita de displasia esquelética, relatório com resultado de outros exames que tenham sido realizados e estudo citogenético nos casos de suspeita de anomalia cromossômica, e/ou molecular (teste de DNA- ácido desoxirribonucleico) ${ }^{(24)}$.

É necessário que se aprimorem os sistemas de informações já disponíveis no Brasil em relação às anomalias cromossômicas, pois esses dados mostram-se bastante escassos. Uma relação maior entre as instituições privadas e as instituições públicas ajudaria muito no sentido de esclarecimento destas informações, disponibilizando dados à população(25).

O estudo aqui apresentado foi realizado a partir de coleta e análise de dados retrospectivos dos prontuários dos nascidos vivos. Ressalta-se que, para o médico, é difícil a identificação exata do motivo da perda de uma gravidez, assim como a prestação de serviços genéticos de alta qualidade, para um bom aconselhamento genético.

No Brasil, foi adotado, a partir do ano 2000, uma versão atualizada da DNV, no Campo 34, a necessidade de preenchimento sobre a presença ou ausência de "malformação congênita e/ou anomalia cromossômica". Este fato é de suma importância para um melhor desenvolvimento da genética comunitária no Brasil. Anteriormente à inserção do Campo 34, as informações eram geradas apenas a partir das Declarações de Óbito (DO), que eram obtidos pelo Sistema de Informação de Mortalidade (SIM) ${ }^{(25)}$.

Apesar deste grande avanço, estudos demonstram que a qualidade da notificação de anomalias congênitas nestes registros nem sempre é satisfatória ${ }^{(25-27)}$. Podemos citar os resultados obtidos por alguns autores ${ }^{(28)}$ que demonstraram um sub-registro de $60,7 \%$ para as malformações congênitas na declaração de nascimento registradas no Sistema de Informação de Mortalidade, obtidas de dados constantes no Sistema de Nascidos Vivos com óbitos em Porto Alegre-RS ${ }^{(28)}$.

Destacamos que a introdução e utilização do Campo 34 apenas terá relevância e confiabilidade com sua implementação de maneira efetiva e correta, com uma grande seriedade do preenchimento dos dados nele contido. Nota-se que muitos fatores comprometem a utilização das DNV como subsídio para informações sobre os defeitos congênitos, um dos principais fatores está relacionado ao momento em que se transcreve a descrição da anomalia do prontuário para a DNV, assim como a utilização de códigos e a alimentação do Sistema de Informações Sobre Nascidos Vivos (SINASC).

Observa-se que, para um melhor estudo das anomalias cromossômicas, há necessidade de melhor aperfeiçoamento dos sistemas de informação já existentes, deste modo seriam evitados erros na interpretação e utilização dos dados relacionados à taxa de insucesso em seu registro. Estratégias para um melhor desempenho nas atividades das ações de saúde, tanto para um atendimento como para a prevenção de doenças genéticas, serão bastante melhoradas quando o preenchimento do Campo 34 for efetivo, trazendo dados com o real valor da realidade do Brasil em relação aos defeitos congênitos.

Os resultados deste estudo refletem de forma generalizada o modo como a DNV é preenchida e principalmente como este documento, de extrema importância para levantamento epidemiológico, tem sido negligenciado em algumas maternidades. Deve-se destacar que a realização de estudo de malformação congênita baseado em dados contidos nas DNVs, torna-se altamente confiável quando possuem o menor número possível de campos em brancos. No entanto, algumas DNVs foram retiradas deste levantamento justamente pelo motivo exposto acima.

É importante destacar o número elevado de DNVs em péssimo estado de conservação, possuindo rasuras ou mesmo ausentes dos prontuários, o que gera uma perda substancial de informações. $\mathrm{O}$ fato de algumas maternidades não possuírem sistema de informatização disponível para alimentar este banco de dados potencializa ainda mais esta perda e dificulta o acesso à informação, fazendo com que a pesquisa seja realizada de forma manual como foi o caso. Por se tratar de uma fonte direta de informação, o cuidado com o preenchimento e conservação das DNVs torna-se de extrema importância, principalmente para geração de perfil epidemiológico e para tomada de decisões em relação a estratégias de prevenção. 
Neste estudo, o principal objetivo foi identificar quais as síndromes cromossômicas estavam presentes na amostra estudada e a sua frequência, para sanarmos a necessidade de gerar algum tipo de dado sobre o assunto no Estado do Amazonas. Sendo assim, após a análise dos prontuários do ano de 2010 a 2014, concluímos que, dentre todas as malformações congênitas registradas em crianças nascidas vivas, 9,2\% eram representadas por anomalias cromossômicas, sendo a mais frequente $(86,6 \%)$ representada pela Síndrome de Down.

Apesar da literatura citar que as aneuploidias tendem a aparecer em conceptos gerados por mães com idade avançada, encontramos prevalências iguais para mulheres de 15-19 e >35 anos. Ressaltamos aqui neste trabalho a grande importância do preenchimento do Campo $34 \mathrm{com}$ acurácia, para que possamos obter o real valor dos dados relacionados à malformação congênita e/ou anomalia cromossômica.

Considerando a carência de estudos sobre anomalias cromossômicas no Estado do Amazonas, este trabalho trouxe resultados descritivos, e o estímulo à sua elaboração foi exatamente para apresentarem dados que possam de alguma maneira colaborar em planos de ação para assistência as famílias e portadores de anomalias cromossômicas, bem como políticas públicas. Acredita-se que os resultados apresentados possam subsidiar o início das discussões para a elaboração de estratégias e políticas públicas adequadas direcionadas a saúde da mulher e da criança no que se diz respeito à prevenção das anomalias congênitas.

Este é o primeiro levantamento de ocorrência e descrição do perfil de nascidos com anomalias cromossômicas, realizado em uma maternidade no Amazonas, que, embora seja o maior Estado da federação brasileira, carece de infraestrutura e pessoal capacitado para realizar este tipo de serviço e atendimento.

\section{REFERÊNCIAS}

1. Santos RS, Dias IMV. Refletindo sobre a malformação congênita. Rev. bras. enferm. 2005;58(5):592-6.

2. Pereira PK, Lima LA, Magnanini MMF, Legay LF, Lovisi GM. Transtornos mentais maternos graves e risco de malformação congênita do bebê: uma metanálise. Cad. Saúde Pública. 2011;27(12):2287-98.

3. Gusmão FAF, Tavares EJM, Moreira LMA. Idade materna e síndrome de Down no Nordeste do Brasil. Cad. Saúde Pública. 2003;19(4):973-8.

4. Ricci SS. Enfermagem materno-neonatal e saúde da mulher. Rio de Janeiro: Guanabara Koogan; 2008.

5. Oliveira1 JV, Westphal F, Abrahäo AR. Impacto do desfecho neonatal em puérperas de recém-nascidos portadores de anomalia congênita. Cogitare Enferm. 2015;20(2):360-7.

6. Castelo e Silva HM, de Almeida KN, Braga MB, Lago EA, Pessoa LTS, da Silva IP. Aspectos fisiológicos e sociais associados às alterações cromossômicas e malformações congênita em gestações perimenopáusicas. Rev Enferm UFPI. 2015;4(1):61-7.

7. Sbragia L. Tratamento das malformações fetais Intraútero. Rev. Bras. Ginecol. Obstet. 2010;32(1):47-54.

8. Dyniewicz AM. Metodologia da Pesquisa em Saúde para Iniciantes. São Paulo: Difusão; 2014.

9. Pierce BA. Genética Essencial: conceitos e conexões. Rio de Janeiro: Guanabara Koogan; 2012.

10. Shawky RM, Sadik DI. Congenital malformations prevalent among Egyptian children and associated risk factors. Egypt J Med Hum Genet. 2011;12(1):69-78.

11. Silva M, Felismino DC, Dantas IC. Malformações fetais: estudo retrospectivo na maternidade da fundação assistencial da Paraíba no município de Campina Grande. Rev. Biol. Ciênc. Terra. 2008;8(1):232-9. 
12. Rio Grande do Sul. Secretaria Municipal de Saúde de Porto Alegre. Pra-Saber: SINASC. [Internet] Porto Alegre; 2002-2003 [acesso em 14 out 2015]. Disponível: http://lproweb.procempa.com.br/pmpa/prefpoa/cgvs/ usu_doc/ev_sinasc_2002_03.pdf.

13. São Paulo. Secretaria Municipal de Saúde de Campinas. Saúde em números/nascidos vivos. [Internet] 2015 [acesso em 11 out 2015]. Disponível: http://www.saude.campinas.sp.gov.br/dados/nasc_vivos/NVOc.htm.

14. de Melo WA, Zurita RCM, Uchimura TT, Marcon SS. Anomalias congênitas: fatores associados à idade materna em município sul brasileiro de 2000 a 2007. Rev. Eletr. Enf. 2010;12(1):73-82.

15. Guerra FAR, Llerena Jr. JC, da Gama SGN, da Cunha CB, Theme Filha MM. Defeitos Congênitos no Município do Rio de Janeiro, Brasil: uma avaliação através do SINASC (2000-2004). Cad. Saúde Pública. 2008;24(1):140-9.

16. Ministério da Saúde (BR). Secretaria de Vigilância em Saúde. Sistema Nacional de Vigilância em Saúde: relatório de situação: Minas Gerais. [Internet] Brasília: Ministério da Saúde; 2005 [acesso em 14 out 2015]. Disponível: http://bvsms.saude.gov.br/bvs/publicacoes/mg_seg.pdf.

17. Mcinnes RR, Nussbaum RL, Willard HF. Thompson \& Thompson Genética Médica. São Paulo: Elsevier; 2008.

18. da Silva RS, de Oliveira CM, Ferreira DKS, do Bonfim CV. Avaliação da completitude das variáveis do Sistema de Informações sobre Nascidos Vivos - Sinasc - nos Estados da região Nordeste do Brasil, 2000 e 2009. Epidemiol. Serv. Saúde. 2013;22(2):347-52.

19. Gardner RJM, Sutherland GR. Chromosome abnormalities and genetic counseling. New York: Oxford University Press; 2004.

20. Magalhães MLC, Furtado FM, Nogueira MB, Carvalho FHC, de Almeida FML, Mattar R, et al. Gestação na adolescência precoce e tardia: há diferença nos riscos obstétricos?. Rev. Bras. Ginecol. Obstet. 2006;28(8):446-52.

21. Senesi LG, Tristão EG, de Andrade RP, Krajden ML, de Oliveira Junior FC, Nascimento DJ. Morbidade e mortalidade neonatais relacionadas à idade materna igual ou superior a 35 anos, segundo a paridade. Rev. Bras. Ginecol. Obstet. 2004;26(6):477-82.

22. Nakadori EK, Soares AA. Síndrome de Down: Considerações gerais sobre a influência da idade materna avançada. Arq. Mudi. 2006;10(2):5-9.

23. Laurino MY, Bennett RL, Saraiya DS, Baumeister L, Doyle DL, Leppig K, et al. Genetic evaluation and counseling of couples with recurrent miscarriage: recommendations of the National Society of Genetic Counselors. J Genet Couns. 2005;14(3):165-81.

24. Ribeiro EM. O pediatra e o aconselhamento genético. Rev Pediatr (Ceará). 2002;3(1):10-7.

25. Nhoncanse GC, Melo DG. Confiabilidade da Declaração de Nascido Vivo como fonte de informação sobre os defeitos congênitos no Município de São Carlos, São Paulo, Brasil. Ciênc. saúde coletiva. 2012;17(4):955-63.

26. de Farias MCAD, Oliveira KMDS, Diniz AS, Maia PCGGS, Valenti VE, de Abrantes KSM, de Abreu LC. Between the capture and dissemination of data: the Importance of the dnv and its adequate completion. Journal of Human Growth and Development. 2014;24(2):150-6.

27. Cechin RVO, Vilela AL, Chaves AA, Martinez JPS, Dias GJN, de Almeida MC, et al. Avaliação do preenchimento correto do campo 34 na Declaração de Nascidos Vivos, na instituição APAE-CAMS I em Cmapo Grande-MS. Ensaios e Ciência: Ciências Biológicas. 2013;17(3):123-33.

28. Cunha J, Aerts D, Leite JCL, Castilla EE. Defeitos congênitos em Porto Alegre: uma investigação da qualidade dos dados registrados na Declaração de Nascido Vivo. Rev. bras. epidemiol. 2002;5(Suppl 1):51. 